\title{
Exploration Into and Practice in Tracking and Monitoring the Tobacco Industry
}

\author{
Li Jinkui ${ }^{1, ~ *, ~ L i ~ T o n g ~}{ }^{2}$, Lu Xiao $^{3}, \mathrm{Wu} \mathrm{Jiarui}^{4}, \mathrm{Xie} \mathrm{Yu}^{1}$ \\ ${ }^{1}$ Training Department Think Tank Research Center for Health Development, Beijing, China \\ ${ }^{2}$ Project Department Think Tank Research Center for Health Development, Beijing, China \\ ${ }^{3}$ Department of Law, Weifang Medical University, Weifang, China \\ ${ }^{4}$ Department of Preventive Medicine, Shanxi Medical University, Xian, China
}

Email address:

Chaihuogun0322@163.com (Li Jinkui),xintanlitong@sina.cn (Li Tong),7402444492@qq.com (Lu Xiao),849534467@qq.com (Wu Jiarui), xieyuthinktank@sina.com (Xie Yu)

*Corresponding author

\section{To cite this article:}

Li Jinkui, Li Tong, Lu Xiao, Wu Jiarui. Exploration Into and Practice in Tracking and Monitoring the Tobacco Industry. Science Journal of Public Health. Vol. 6, No. 1, 2018, pp. 15-20. doi: 10.11648/j.sjph.20180601.13

Received: October 17, 2017; Accepted: October 17, 2017; Published: December 5, 2017

\begin{abstract}
To keep abreast of strategies and actions of the tobacco industry to interfere in tobacco control and take effective measures. Monitoring tobacco industry related news, websites, social media and publications in an all-round way and watching tobacco policies, marketing, promotion and other activities that interfere in tobacco control closely. Having been engaging in tracking and monitoring the tobacco industry for eight years, Think Tank Research Center for Health Development has timely disclosed the tobacco industry's activities that went against the Framework Convention on Tobacco Control. We worked with public interest lawyers and made complaints to related legal authorities, as a result, illegal activities of the tobacco industry have been stopped, investigated and dealt with. Relative information has been publicized and spread through media to expose and crack down on all activities minimizing harm of smoking and misleading the public. Think Tank Research Center's exploration into and practice in tracking tobacco are comprehensive, documentary, timely and well directed. Tracking and monitoring of our civil institution have strongly fought back against the tobacco industry's anti-tobacco control activities! We call on that a national-wide, all-inclusive and systematic monitoring system should be established for the tobacco industry.
\end{abstract}

Keywords: Tobacco Tracking, Monitoring, Viewpoint of the Public, Media Release, Disclosure,

Monitoring Information Database

\section{Introduction}

Article 5.3 in the Framework Convention on Tobacco Control (hereinafter referred to as Convention) of WTO pointed out, "When making and practicing public health policies in the aspect of tobacco control, all contracting parties shall act according to national laws to prevent the policies from being affected by commercial and other vested interests of the tobacco industry". Interference of the tobacco industry is the biggest threat to health policies. Given the fundamental and irreconcilable conflicts between its interests and public health policies, the tobacco industry surely tries to stand in the way of effective tobacco control policies. WTO pointed out that the tobacco industry had tried to take various forms of interference in the implementation of effective tobacco control policies, including kidnapping political and legislative process, overstating the industry's importance in economy, manipulating public opinion, falsifying support through frontier groups, slandering scientific evidence about tobacco and second-hand smoke and threatening the government with accusation or threatening to sue the government [1]. The 2012 World No Tobacco Day Theme Report jointly released by ThinkTank Research Center for Health Development, Chinese Association on Tobacco Control and Chinese Preventive Medicine Association summarized Chinese tobacco industry's measures to interfere in tobacco control in the following eight aspects: 1) Minimizing legally binding force of the Convention to make the government's "political commitment" 
impracticable; 2) Denying scientific evidence about the harm of tobacco to promote sustainable growth in tobacco sales volume; 3) Cheating the public with the strategic path of "harm reduction - tar reduction - flavoring"; 4) The tobacco industry's been trying to prevent health warning charts from being printed on cigarette packaging; 5) The tobacco industry's "three reasons" and "one countermeasure" in coping with cigarette tax price strategy; 6) Promoting tobacco consumption through hidden ads, promotion and sponsorship; 7) Crazy high-end cigarettes and 8) Roping teenagers in with tobacco and strengthening their smoking aspirations $\mathrm{t}$ [2].

Article 20.4 in the Convention put forward explicit requirements on monitoring the tobacco industry, emphasizing that all contracting states should collect and exchange "information related to the Convention, including the tobacco industry's activities and tobacco planting" t [3]. Article 20.4c of the Convention also pointed out that all contracting states should "cooperate with related international organizations, gradually building and maintaining the global system, collecting and spreading the tobacco industry's related activity information that affects tobacco production, processing and the Convention or national tobacco control activities on a regular basis" t [3]. The 2008 WTO Global Tobacco Epidemic pointed out that an excellent tobacco monitoring system must carry out monitoring on tobacco enterprises' marketing, promotion and lobbying as well. Only by fully understanding the tobacco industry's strategies and actions for interfering in tobacco control, can we take effective countermeasures. Therefore, monitoring the tobacco industry's related activities seems to be of paramount importance.

Performing the Convention has become the whole society's public welfare action led by the government. Tobacco control means reducing demand and supply of tobacco. However, the gap between situations of tobacco production, supply, advertising, sponsoring, marketing, identification of tobacco packaging and tax price in China and requirements on fulfilling tobacco control is rather wide. Thus, collection, exchange of, and comment on information related to the tobacco industry's performance of the Convention and release of the information to all social circles to strengthen information exchange are necessary to promote tobacco control and performance of the Convention in China. At present, monitoring on the tobacco industry and its activities has been launched in China. These monitoring activities include the Tobacco Tracking Briefing of ThinkTank Research Center for Health Development, public opinion monitoring on tobacco control and the tobacco industry's activities jointly launched by Chinese Center for Disease Control and Prevention, Union Medical College and Campaign for Tobacco-Free Kids as well as coverage of the tobacco industry's activities on Chinese Association on Tobacco Control website.

As a civil institution on tobacco control, ThinkTank Research Center for Health Development has been working to advocate tobacco control in China for many years. We have felt deeply the negative impact of the tobacco industry's activities on performing the tobacco control convention.
Therefore, we have paid close attention to, explored into and practiced in tracking and monitoring Chinese tobacco industry's activities as well as edited the Tobacco Tracking Briefing for the reference of enthusiastic persons who cared about tobacco control.

\section{Purposes}

The Tobacco Tracking Briefing aims at tracking situations and information of the tobacco industry in such aspects as policies, leadership trends, production, sales, tobacco culture and enterprise social responsibility; tracking the competent government departments' measures and actions in performance; observing and commenting on tobacco enterprises' activities that violate the Convention while focusing on reflecting new situations, trends and problems related to tobacco control from both sides so as to report the latest dynamics of tobacco to people that are enthusiastic in tobacco control. We do our best to be well-grounded, objective and rational and analyzing things comprehensively and in-depth.

\section{Basis}

\subsection{Article 5.3 of the Convention}

When making and practicing public health policies in the aspect of tobacco control, all contracting parties shall act according to national laws to prevent the policies from being affected by commercial and other vested interests of the tobacco industry.

\subsection{Article 5.3 Implementation Criteria of the Convention}

"The fundamental and irreconcilable conflicts between the tobacco industry's interests and public health policies".

\subsection{Requirement in Article 8 Implementation Criteria 46 of the Convention}

"Monitoring and evaluation of measures that reduce contact of tobacco smoke are important".

\subsection{Requirements of the MPOWER Strategy}

Monitoring the usage of tobacco and preventive policies Provide accurate national and global tobacco usage data Indicate tobacco's impact on public health and economy Measure the effectiveness of tobacco control policies

Expose the tobacco industry's strategies in terms of marketing, promotion and lobbying

In the institution, Investigate the employees and track the effects of the policies.

\section{Contents}

Tobacco advertisement, promotion and sponsorship activities (including "enterprise social responsibility activities" of tobacco enterprises and anti-tobacco control activities in the 
name of tobacco control);

Scientific research activities, research funds and awards for science and technology of the tobacco industry (including research activities and related false propaganda of so-called "low hazard cigarette");

Related policies, production and sales of tobacco, import, export and financial situations of the tobacco industry;

Tax and price change of cigarettes;

Legal proceedings related to the tobacco industry;

Mouthpiece of the tobacco industry, including scientific research personnel and news media;

Other activities of the tobacco industry, e.g., monitoring on related coverage of illegal trade.

\section{Methods}

\subsection{Network Monitoring}

Major portals, sites of Tobacco Monopoly Administrations at state and provincial levels and major tobacco companies are monitored at a frequency of once every three days at least. Major contents of the sites shall be read through and important information extracted.

\subsection{Monitoring on Related Publications of the Tobacco Industry}

Yearbook, annual report and important publications of the tobacco industry shall be read through and important information extracted.

\subsection{Monitoring on Trend of Scientific Research of the Tobacco Industry}

Through Cnki. net, research papers of the tobacco industry in related domestic professional periodicals and sites of domestic scientific research institutions are monitored and the major research direction analyzed.

\section{Edit and Distribution}

Tobacco Tracking Briefing that is edited by ThinkTank Research Center for Health Development invites professional commentators to evaluate key information. Albums that carry out in-depth monitoring on and analysis of focusing events (e.g. "harm reduction and tar reduction" and "tobacco academician") of the tobacco industry are published when necessary. Electronic edition of the briefing is on the homepage of the China Tobacco Control Resource Center website for professionals majoring in tobacco control to download and print editions are sent to related professional tobacco control institutions and personnel, including health education workers all over the country, medical, public health, legal and economic experts, academies, associations, media and people who are enthusiastic in tobacco control. The Brief was first published in August, 2008. As a quarterly publication, by January, 2017, it has published totally 28 editions and 5 special albums (including Harm Reduction and Tar Reduction Album, Who is marketing death? We Never Give Up-Forbid
All Tobacco Ads, Promotion and Sponsorship, Disaster-If the 5.4 Million Tobacco Shops Are Allowed to Advertise and Be Aware of the Interference from the Tobacco Industry) and 30 editions of press news. The total circulation was about 15,000.

\section{Tracking Hot Spots}

\subsection{Chinese Tobacco Industry Laid Down the "Sustainable Development Strategy” to Confront Performance Head on}

Ten years after Chinese Government signed the Convention, instead of reducing supply and demand that was stipulated in the Convention, Chinese tobacco industry has increased sales volume of cigarettes year by year. It was the rapid growing profits that propelled the industry to carry out the "sustainable development strategy" firmly.

\subsection{Chinese Tobacco Industry's "Tar Reduction and Harm Reduction Strategy"}

The tobacco industry announced that the choice of tar reduction and harm reduction was the most scientific, rational, responsible and practical solution for the tobacco industry presently.

\subsection{Strategy Shift of Chinese Tobacco Industry}

The shift from tar reduction and harm reduction to harm reduction and tar reduction was the result brought about by the adopted so-called "hazard assessment model", which lowered some hazardous compositions in tobacco smoke selectively.

\subsection{Chinese Tobacco Industry's "Strategy of Preventing Warning Signs from Being Printed on Cigarette Packaging”}

Chinese tobacco industry has explicitly indicated that it would "meet cigarette packing warning requirements based on national conditions". The Provisions of Cigarette Packaging Labeling in People's Republic of China jointed released by State Tobacco Monopoly Administration and General Administration of Quality Supervision, Inspection and Quarantine of the People's Republic of China in 2007 regulated that warning signs on cigarette packing shall be implemented according to the principle of "low limit performance, limit visibility and no pictures". The industry firmly opposed to printing warning signs on cigarette packing with the excuse that the Convention "did not regulated picture warnings compulsively; most contracting parties did not have picture warnings on cigarette packing".

\subsection{Tobacco Advertisement, Promotion and Sponsorship}

The existing Advertising Law expressly prohibits tobacco ads in five media and four places. However, illegal tobacco ads are seen everywhere; the tobacco industry makes use of loopholes of the law in all types of promotion and marketing wantonly and has set its eye on emerging media; in the name of social responsibility of enterprises, it conducts charity 
sponsorship, tobacco brand naming, sponsoring poor students and sports and culture activities.

\subsection{Tax and Price Are Not Interconnected}

Prices paid behind the high profits and taxes of the tobacco industry: Every year, hundreds of thousands of people die of smoking-related diseases. Carpet smoking ban in environment is interfered in with the harmless theory of second-hand smoke; profits and taxes of the tobacco industry have lagged far behind the social economic burden caused by harm of tobacco.

\subsection{Integration of Government and Enterprises}

How does the system of integration of government and enterprises in the tobacco industry impact on the promulgation and practice of national control policies?

\subsection{Trends in the Leadership of the Tobacco Industry}

Surveys and conference speeches of leaders in the tobacco industry: Leaders at ministerial and provincial levels encourage and take part in tobacco-related activities.

\subsection{Tobacco Cultural}

Tracking and disclosing marketing activities of the tobacco industry carried out in the name of culture.

\subsection{Lawsuit of Tobacco}

Game actions of tracking tobacco control and tobacco control.

\section{Spread of Actions and Results}

ThinkTank Research Center has been tracking activities of the tobacco industry that violated the Convention, stopping and investigating multiple illegal activities of the industry through timely disclosure, complaint and application from the civil perspective and releasing and spreading them through media. Examples are as follows:

\subsection{Journal Output}

Based on tracking of the tobacco industry, we edited and issued four editions of Observation of Tobacco Control in China-Public Point of View; we compiled the book-Whose Interest Is the Chinese Tobacco Industry Fighting for based on its Two Countermeasures to cope with the Convention t [4]. We wrote the 2012 World No Tobacco Day Theme ReportMind the Interference for the Tobacco Industry for the theme of 2012 World No Tobacco Day. On the 2013 World No Tobacco Day, we released the tobacco tracking album-Who is marketing death? by tracking, monitoring and collecting examples and pictures of tobacco ads, promotion and sponsorship of the tobacco industry.

\subsection{Spread of Results}

Several press conferences were held in the name of
ThinkTank Research Center for Health Development annually to spread monitoring results widely. Besides, press conferences were held in time to release any accidental hotspot and focusing event found in the monitoring.

\subsection{Establishment of the Monitoring Information Database of the Tobacco Industry}

A professional website on tobacco industry monitoring was established: Original information was uploaded to the website of China Tobacco Control Resource Center in categories. An internal search engine was established for people who were enthusiastic in tobacco control. On the homepage, an important initiative column of the tobacco industry was set to spread emergencies and important information rapidly.

\subsection{The "Blue Trend Runs for Love" Activity Was Punished}

In 2010, the big event of " 2010 Zhongnanhai Love Convey Activity- Blue Trend Runs for Love" was jointly held by the Beijing Donation Center of Hope Project of Beijing Youth Development Foundation and the "Zhongnanhai Love Fund" of Beijing Cigarette Factory. We learned that the background plates, clothes and props on the meeting site of the activity carried tobacco ads, so we complained to the Chaoyang Branch of Beijing Administration for Industry and Commerce and made an application. As a result, the activity was decided to be illegal and punished.

\subsection{Tobacco Characteristics Were Removed from Hope Schools Built by Tobacco Enterprises}

In 2012, ThinkTank Research Center found out that a few "Hope Schools" in Sichuan that were named after tobacco brands and words had tobacco enterprises' signs on their building roofs and placards saying "Genius stems from diligence and tobacco helps you to become a useful person" on campus. We complained to the Sichuan administration for industry and commerce. Finally, the tobacco characteristics were removed from the schools.

\subsection{Disclosure of the Tobacco Industry's Fraud of Harm Reduction and Tar Reduction}

All scientific studies have shown that changes in cigarette design, including filters, low tar and light flavour, did not reduce the overall risk of disease among smokers, but might hamper smoking prevention and smoking cessation efforts.

\subsection{Disclosure of the Tobacco Industry's “Cultural Marketing Strategy"}

In Oct., 2013, Think Tank Research Center held a meeting to disclose the fact that cultural marketing of the tobacco industry was a kind of "unarmed counter move" and behavior that spread social negative energy. We suggested that health warning signs should be printed on cigarette packings, tobacco ads, promotion and sponsorship in all forms prohibited and marketing activities of the tobacco industry carried out in the name of culture opposed. 


\subsection{Complaint About China Tobacco Jiangxi Industrial LLC and China Tobacco Guangdong Industrial LLC}

In March, 2013, ThinkTank Research Center found that there were a large amount of promotional text and pictures that violated the Advertising Law t [5] and Interim Measures for the Administration of Tobacco Advertisements on the official websites of China Tobacco Jiangxi Industrial LLC and China Tobacco Guangdong Industrial LLC. We complained timely and received replies of treatment correspondingly.

\subsection{Complaint About the Illegal Tobacco Advertisement in Southern People Weekly}

Complaint about the illegal tobacco advertisement on iamsharer.com and yanyue.cn;

Complaint about the illegal tobacco advertisement issued in the activity of "2013 China International West Lake \& Da Hong Ying Rose Wedding" by China Tobacco Zhejiang Industrial LLC;

Complaint about the tobacco advertisement published in Kunming Metropolitan Times.

\section{Conclusions and Outlook}

\subsection{Characteristics}

Tracking and monitoring in the Tobacco Tracking Briefing over the past eight years all had following features:

\subsubsection{Comprehensiveness}

Comprehensive information including both statement of fact and comments; Illustrated with caricatures and blog article selection (e.g., An Old Smoker Talks about Cigarettes);

\subsubsection{On-the-spot Record}

Stating the fact by reprinting materials that have been released by media, tobacco control-related tobacco production, supply, advertising and marketing in China released on the tobacco industry's websites mainly. Each down-to-earth message was marked with source and general arrangement.

\subsubsection{Timeliness}

The quarterly publication followed the trend of tobacco at all time and took corresponding measures and actions in time;

\subsubsection{Pertinence}

According to articles in the Convention, we reviewed the facts that the tobacco industry had violated the Convention. The more powerful analysis made the public polish their eyes.

\subsection{Outlook}

Currently, though many units started and joined the monitoring on Chinese tobacco industry, no all-inclusive and systematic monitoring system has been formed and a lot of related hotspot issues have not been found and spread. For instance, the 2012 "tobacco academician" and the "construction and application of the theoretical system of cigarettes with Chinese characteristics" that participated in the selection of National Science and Technology Award were both found by civilians first [7]. So it should be one of China's recent focuses in tobacco control to integrate all forces to establish an all-inclusive and systematic monitoring system for the tobacco industry.

In 2013, ThinkTank Research Center for Health Development and Chinese Center for Disease Control and Prevention cooperated to research and made the Monitoring Scheme of Tobacco Industry in China, aiming at exploring to establish an all-inclusive and systematic monitoring system for the tobacco industry that monitored the industry's marketing, promotion, lobbying and other activities that stood in the way of tobacco control in China in an all-round way, so that timely actions could be made to fight back its interference. Through monitoring on the tobacco industry, establishment of a national-wide monitoring team was planned to strengthen tobacco control ability of the national health education system and the disease control system.

One of the tobacco control measures in the Tobacco Control Plan in China (2012-2015) released in Dec., 2012 was to establish the tobacco epidemic monitoring information system in China [6]. The measure stated that international standards and practices should be borrowed to establish a unified, formal and authoritative tobacco epidemic monitoring system; special investigations and studies should be launched and the investigation content of tobacco usage be added to residents health survey items. Smoking rate of groups of different genders, ages and occupations in various regions shall be grasped exactly, demographic, behavioral and psychological characteristics of tobacco consumers fully understood, trends in tobacco epidemic, consumption modes, influence factors and consequences scientifically forecasted and tobacco hazard research strengthened to provide systematical, comprehensive, accurate and reliable information support for making tobacco control policies and assessing tobacco control effect. In 2012, the central subsidized local health quality promotion action project was launched. The project carried out monitoring on health quality and tobacco epidemic in 31 provinces (districts and cities) and 336 monitored counties (cities and districts) throughout the country. A national-wide health quality and tobacco epidemic monitoring system would be established gradually [8].

In 2015, the central grants for local health literacy promotion project will continue to carry out a nationwide large-scale population survey, in order to obtain national and provincial health literacy and tobacco epidemic monitoring data, and provide scientific basis for governments and health and family planning administrative departments to formulate relevant policies [9], Comprehensively implement the outline of "healthy China 2030" and further promote the implementation of tobacco control [10].

\section{References}

[1] 2012. WTO. Interference of the tobacco industry in tobacco control. Geneva; 2012. 
[2] ThinkTank Research Center for Health Development, Chinese Association on Tobacco Control and Chinese Preventive Medicine Association. 2012 World No Tobacco Day Theme Report. Beijing; 2012.

[3] WTO Framework Convention on Tobacco Control.

[4] WHO "the Framework Convention on tobacco control case and countermeasure research of China tobacco. Economic Science Press. 2006.

[5] Advertisement Law of the People's Republic of China.

[6] China tobacco control plan (2012-2015 years).
[7] 'Tobacco Academician' was not invite to attend the 11th Academician Congress of CAE, CAE will continue to discuss his qualification. South Metropolis Daily 2012. http://gcontent.oeeee.com/1/49/14919153f8aaf3af/Blog/f04/52 69ac.html (accessed31 Jan 2013).

[8] Chinese Center for Disease Control and Prevention. Global Adults Tobacco.

[9] Health literacy of Chinese citizens - basic knowledge and skills (2015 Edition.).

[10] Outline of "healthy China 2030". 2016. 